\title{
Morphology and capping effects in the magnetic and magneto-optical properties of nanoparticulate Co films
}

\author{
C. Clavero, ${ }^{1,2}$ L. Martínez, ${ }^{3}$ A. García-Martín, ${ }^{1}$ J. M. García-Martín, ${ }^{1}$ Y. Huttel, ${ }^{3}$ N. D. Telling, ${ }^{4}$ G. van der Laan, ${ }^{4,5}$ \\ A. Cebollada, ${ }^{1}$ and G. Armelles ${ }^{1}$ \\ ${ }^{1}$ Instituto de Microelectrónica de Madrid IMM-CNM-CSIC, Isaac Newton 8 (PTM), Tres Cantos, 28760 Madrid, Spain \\ ${ }^{2}$ Department of Applied Science, College of William and Mary, McGlothlin-Street Hall, P.O. Box 8795, \\ Williamsburg, Virginia 23187, USA \\ ${ }^{3}$ Instituto de Ciencia de Materiales de Madrid ICMM-CSIC, C/Sor Juana Inés de la Cruz 3, Cantoblanco, 28049 Madrid, Spain \\ ${ }^{4}$ Magnetic Spectroscopy Group, Daresbury Laboratory, Warrington WA4 4AD, United Kingdom \\ ${ }^{5}$ Diamond Light Source, Chilton, Didcot, Oxfordshire OX11 ODE, United Kingdom
}

(Received 26 October 2007; revised manuscript received 16 December 2007; published 17 March 2008)

\begin{abstract}
Magnetic and magneto-optical properties of Co films are studied as a function of the morphology and the capping layer. We show that the nanoparticulate structure of the Co films has a clear influence on the magnetic and magneto-optical properties of the system. Kerr measurements combined with x-ray magnetic circular dichroism provide evidence of a strong correlation between the collective magnetic behavior of the system and the individual atomic magnetic response. The influence of the magnetic nature of the capping layer (Al, Au, and $\mathrm{Pt}$ ) is also analyzed. Polarized capping layers, such as $\mathrm{Pt}$, magnetically couple the nanostructures and not only increase the effective anisotropy of the system but also enhance the atomic magnetic moment of Co and the global magneto-optical activity.
\end{abstract}

DOI: 10.1103/PhysRevB.77.094417

PACS number(s): 75.70.-i, 78.70.Dm, 78.67.-n

\section{INTRODUCTION}

Magnetic nanoparticles have generated growing interest as their restricted dimensions result in a drastic change in the physical properties of the system. In particular, nanoparticles of transition metals such as cobalt, iron, or nickel are highly magnetic with moments that exceed the bulk values. ${ }^{1-3}$ The reduced coordination of the atoms at the surface is one of the reasons of this behavior since it enhances the orbital magnetic moment. ${ }^{4,5}$ The examination of the influence of different physical parameters on the spatial and magnetic order in nanoparticulate systems should contribute to a better understanding of the nature of the interactions responsible for the magnetism. ${ }^{1}$ This in turn will enable the magnetic properties of these materials to be tailored to specific applications, such as for magnetic recording.

Another important feature to take into account, in order to achieve the desired modified magnetic response, is the nature of the materials in contact with the magnetic system. In nanoparticulate systems, the high fraction of atoms at the surface critically enhances the role of the interface. ${ }^{7}$ Thus, the capping layer is not only a key factor to preserve the stability of the magnetic system but also influences the magnetic response through hybridization with the $3 d$ orbitals of the transition metals. ${ }^{6}$ This makes it important to study changes in the magnetic properties of nanoparticles due to coating with different materials. ${ }^{8}$

In this paper, we report the effect of three metal capping layers with different tendencies for induced magnetic polarization. These are Pt, which shows a strong tendency to be magnetically polarized, $\mathrm{Au}$, which only weakly polarizes, and $\mathrm{Al}$, which does not polarize. A complete understanding of these systems is a challenging issue with potential applications in high-density data storage devices and magnetooptical sensors. ${ }^{9}$ We examine the influence of both the nano- particulate structure and the capping layer on the magnetic and magneto-optical properties. Kerr measurements are performed to study the collective magnetic behavior of each system. This magneto-optical characterization is highly sensitive to small amounts of material and to modifications of the morphology and electronic structure of the system. ${ }^{7}$ These results are complemented with $\mathrm{x}$-ray magnetic circular dichroism (XMCD) measurements that are able to extract the element-specific local magnetic moments. ${ }^{10}$ This technique enables a better understanding of the magnetic properties, as it is an atomiclike probe of the magnetism.

\section{EXPERIMENTAL DETAILS}

The sample preparation was performed by means of sputtering deposition in an ultrahigh-vacuum chamber with base pressure in the low $10^{-9}$ mbar range. $1.2 \mathrm{~nm}$ thick Co films were deposited on $\mathrm{Al}_{2} \mathrm{O}_{3}(0001)$ substrates by magnetron sputtering deposition at $1.5 \times 10^{-4} \mathrm{mbar} \mathrm{Ar}$ and with three different substrate temperatures applied during the Co deposition [namely, room temperature (RT), $300{ }^{\circ} \mathrm{C}$, and $600{ }^{\circ} \mathrm{C}$ ] in order to promote a two-dimensional (2D) to threedimensional (3D) growth mode transition, as has been observed in similar systems. ${ }^{8}$ A low voltage of $450 \mathrm{~V}$ was applied to the Co target ( $4 \mathrm{~W}$ sputtering power) in order to obtain a slow and well controlled Co deposition rate of $0.032 \AA / s$. Following Co deposition, three different metallic layers (namely, $\mathrm{Au}, \mathrm{Al}$, and $\mathrm{Pt}$ ) with thicknesses of $3.5 \mathrm{~nm}$ were deposited on top by means of triode sputtering deposition at $4 \times 10^{-4}$ mbar Ar, with deposition rates of $0.5,0.32$, and $0.21 \AA / \mathrm{s}$, respectively, and at RT in order to promote a conformal growth mode with the Co surface.

The sample structure and morphology were characterized by atomic force microscopy (AFM) and $\mathrm{x}$-ray techniques such as X-ray reflectrometry (XRR) and x-ray diffraction 
(XRD). AFM images were taken using a Nanotec ${ }^{\mathrm{TM}}$ microscope operating in noncontact dynamic mode, i.e., with a mechanically modulated cantilever. Silicon cantilevers from NanoSensors ${ }^{\mathrm{TM}}$ with a force constant $k \approx 5 \mathrm{~N} / \mathrm{m}$ and tip radius of $\sim 10 \mathrm{~nm}$ were used. The XRD and XRR experiments were performed in a four-circle diffractometer with $\mathrm{Cu} K \alpha$ $(\lambda=1.5418 \AA)$ radiation using a Bragg-Brentano configuration and $1 / 4^{\circ}$ slits.

The magnetic characterization was carried out by means of Kerr effect techniques and XMCD. In particular, in situ transverse Kerr loops were measured at RT by applying an in-plane magnetic field and using a $633 \mathrm{~nm} \mathrm{HeNe}$ laser at $45^{\circ}$ incidence angle geometry. Loops were measured in situ before and after the capping layer deposition in order to study its influence on the magnetic behavior. In the case of the ex situ polar Kerr loops, $530 \mathrm{~nm}$ light in normal incidence was used and the Kerr rotation angle was recorded. The magneto-optical (MO) activity of the samples was studied by means of a polar Kerr spectrometer in the spectral range from 1.4 to $4.3 \mathrm{eV}$. Details of the technique can be found elsewhere. ${ }^{11}$ The magnetic characterization of the samples with XMCD was performed by x-ray absorption spectroscopy (XAS) in total electron-yield mode on beamline 5U.1 of the Synchrotron Radiation Source (SRS) at Daresbury, UK. All XMCD measurements were performed at RT using an eight-pole vector magnet which can provide magnetic fields up to $\pm 0.9 \mathrm{~T}$. The results presented here were measured while applying the magnetic field parallel to the incident $\mathrm{x}$-ray beam and reversing its direction at each photon energy point. The degree of polarization was 53\% as deduced from a reference sample with the structure $30 \AA$ $\mathrm{Al} / 40 \AA \mathrm{Co} / \mathrm{Al}_{2} \mathrm{O}_{3}(0001)$. Incomplete magnetization saturation in the XMCD geometry was corrected with the magnetization values extracted from the Kerr hysteresis loops measured in the polar configuration.

\section{III. $\mathrm{Co} / \mathrm{Al}_{2} \mathrm{O}_{3}$ SYSTEM}

For this study, commercial $\mathrm{Al}_{2} \mathrm{O}_{3}(0001)$ substrates with a measured miscut angle of $3.25^{\circ}$ were used. XRD measurements revealed that the miscut direction had an angle of $45^{\circ}$ with the in-plane principal crystallographic direction [1120] of the $\mathrm{Al}_{2} \mathrm{O}_{3}(0001)$ lattice. This results in the formation of terraces of atomic height on the surface, whose edges are aligned along different directions, as can be observed in the AFM image in Fig. 1. XRD measurements of a $25 \mathrm{~nm}$ thick Co film grown on the hexagonal $\mathrm{Al}_{2} \mathrm{O}_{3}(0001)$ face showed that the Co grows as an hexagonal closed packed (hcp) phase, with its lattice rotated $30^{\circ}$ with respect to that of the substrate.

Previous reports on similar magnetic systems indicated the presence of in-plane uniaxial anisotropies related to the existence of monatomic-height steps. For example, uniaxial magnetic anisotropies with the easy axis perpendicular to the step edges were found in $\mathrm{Fe} / \mathrm{W}(100),{ }^{12,13}$ whereas anisotropies with the easy axis parallel to the step edges were found in $\mathrm{Co} / \mathrm{Cu}(100),{ }^{14-16} \mathrm{Fe} / \mathrm{Ag}(100),{ }^{17}$ and $\mathrm{Co} / \mathrm{Au}(322) .{ }^{18} \mathrm{In}$ order to investigate the in-plane magnetic anisotropy in the

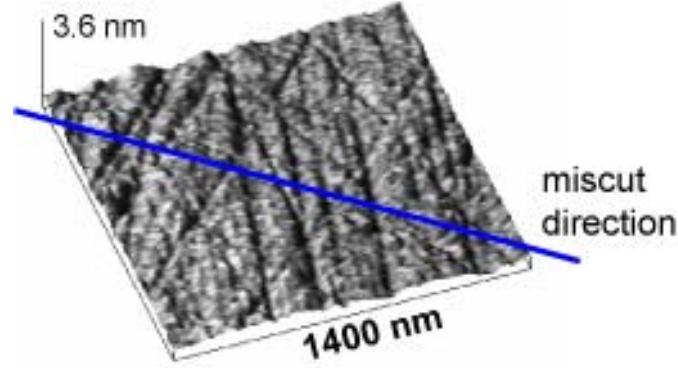

FIG. 1. (Color online) AFM image for the bare $\mathrm{Al}_{2} \mathrm{O}_{3}(0001)$ substrate, in which atomic height terraces are visible. The drawn blue line represents the miscut direction of the substrate. Image size: $3.6 \mathrm{~nm}$ vertical and $1400 \mathrm{~nm}$ lateral.

$\mathrm{Co} / \mathrm{Al}_{2} \mathrm{O}_{3}$ system, in situ transverse Kerr loops were obtained for the abovementioned $25 \mathrm{~nm}$ thick Co film (Fig. 2), with the magnetic field applied along different in-plane directions. The magnetization reversal processes were found to depend on the in-plane direction. For instance, at $90^{\circ}$ to the principal in-plane direction $[11 \overline{2} 0]$, a square loop with a single switching field is found, whereas at $150^{\circ}$ to [11 $\left.\overline{2} 0\right]$, three switching fields are detected, revealing the existence of a complex magnetization reversal process. Figure 2 also shows the reduced in-plane magnetic remanence $\left(M_{r} / M_{s}\right)$ as a function of the applied field angle. As can be seen, the magnetization exhibits a nonsymmetric shape, suggesting the existence of several competing in-plane magnetic anisotropies. To explain the origin of these anisotropies, several factors must be taken into account. First, the atomic height terraces of the substrate are aligned along different directions (as shown by AFM), giving rise to a complex step-induced magnetic anisotropy. Second, the orientation of the Co lattice

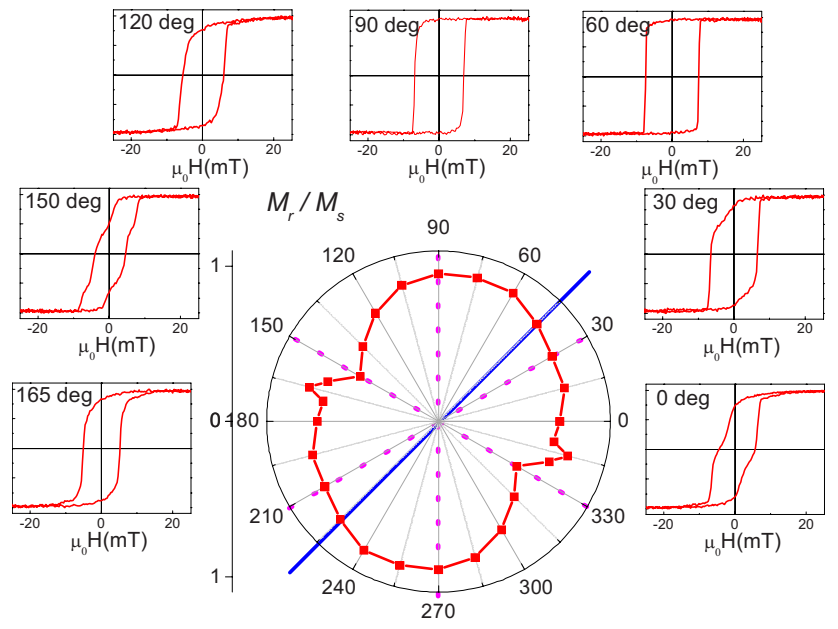

FIG. 2. (Color online) Transverse Kerr loops measured for a $25 \mathrm{~nm}$ thick continuous Co film with the applied magnetic field along different in-plane directions, as indicated. Center: polar plot showing the reduced remanence magnetization $\left(M_{r} / M_{s}\right)$ versus the angle of the applied field. The misfit direction (perpendicular to the average terrace edges direction) is shown by the drawn blue line, and the three principal crystallographic directions of the Co film are shown by the dotted lines. 


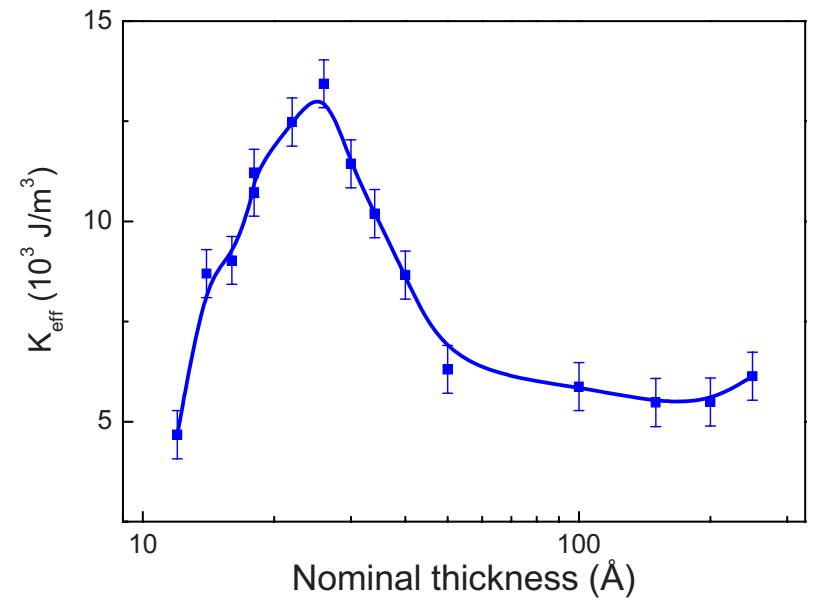

FIG. 3. (Color online) Effective magnetic anisotropy constant $\left(K_{\text {eff }}\right)$ as a function of the Co thickness. The line is a guide to the eye.

may introduce an additional crystallographic anisotropy. Owing to the fact that the shape of the $M_{r} / M_{s}$ plot (Fig. 2) is closer to that of a uniaxial anisotropy system, it can be deduced that the step-induced anisotropy is the stronger contribution in this case. It can also be seen that the in-plane easy axis is nearly perpendicular to the average terrace edges direction, which is consistent with the $\mathrm{Fe} / \mathrm{W}(100)$ system.

Additionally, the value of the magnetic anisotropy as a function of the Co thickness was investigated to confirm its mainly step-induced origin. The effective anisotropy constant $\left(K_{\text {eff }}\right)$ of Co films of thicknesses up to $25 \mathrm{~nm}$ were obtained from the saturated transverse loops measured along the hard axis, and its dependence with the nominal Co thickness is shown in Fig. 3. An initial increase of $K_{\text {eff }}$ was observed for Co thickness between 1.2 and $2.6 \mathrm{~nm}$, reaching a maximum value of $1.35 \times 10^{-4} \mathrm{~J} / \mathrm{m}^{3}$, probably related to a nonperfect magnetic connection between the Co deposited on the different terraces. No in situ transverse MO Kerr signal was obtained for thickness below $1.2 \mathrm{~nm}$ due to the detection limit of the experimental setup. For higher Co thickness, a progressive and slow decrease of $K_{\text {eff }}$ was observed, revealing a weak dependence with thickness, as reported previously for $\mathrm{Fe} / \mathrm{W}(100)$ (Ref. 12) and $\mathrm{Fe} / \mathrm{Ag}(100)$ (Ref. 17) systems. Berger et al. ${ }^{14}$ and Choi et al. ${ }^{12}$ ascribed this step-induced magnetic anisotropy to the effect of lattice symmetry breaking in the steps and to the influence of strain at the step corners. They concluded that the direction of the induced uniaxial anisotropy (parallel or perpendicular to the edges of the terrace direction) depends on the substrate-film electronic hybridization.

\section{MORPHOLOGY}

The surface morphology of nominally $1.2 \mathrm{~nm}$ thick Co films grown at RT, $300{ }^{\circ} \mathrm{C}$, and $600{ }^{\circ} \mathrm{C}$, before and after $\mathrm{Al}$, $\mathrm{Au}$, and Pt capping layer deposition, was investigated by means of AFM. First, in order to study the morphology evolution as a function of the Co deposition temperature, ex situ AFM images were obtained for a series of uncapped Co (a) $\mathrm{Al}_{2} \mathrm{O}_{3}$

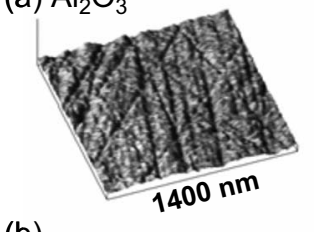

VERTICAL SCALE:

$3.6 \mathrm{~nm}$ in all the images

(b)
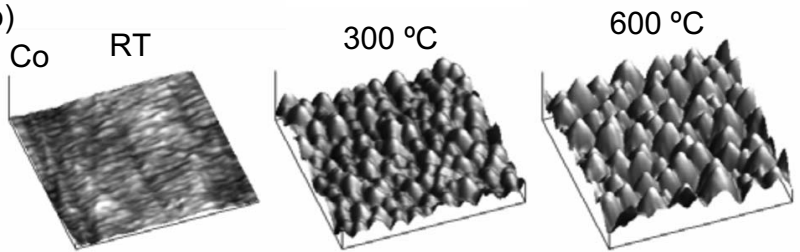

Al
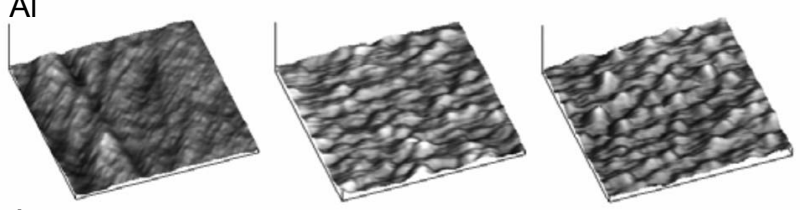

$\mathrm{Au}$
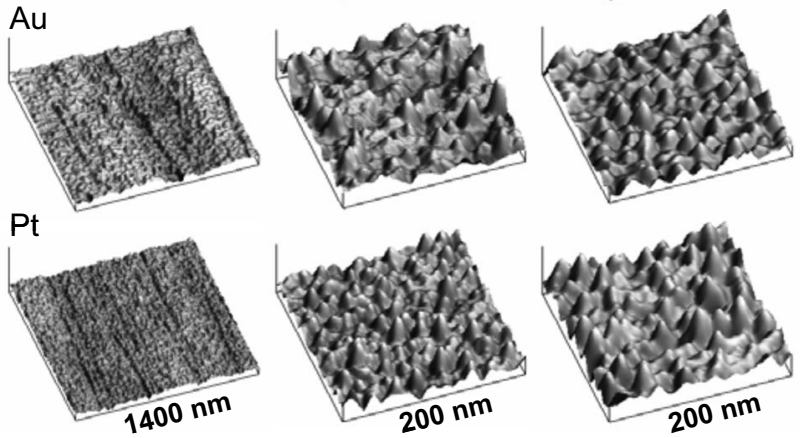

FIG. 4. (a) AFM image for the $\mathrm{Al}_{2} \mathrm{O}_{3}(0001)$ substrate. (b) $\mathrm{AFM}$ images for the Co films grown at RT, $300{ }^{\circ} \mathrm{C}$, and $600{ }^{\circ} \mathrm{C}$ (columns), uncapped and capped with $\mathrm{Al}, \mathrm{Au}$, and Pt films (rows). The vertical scale is $3.6 \mathrm{~nm}$ in all images.

films grown at RT, $300{ }^{\circ} \mathrm{C}$, and $600{ }^{\circ} \mathrm{C}$ [Fig. 4(b)]. In order to minimize the effect of Co surface oxidation, the AFM measurements were performed immediately after the samples were removed from the UHV chamber. The film deposited at RT reproduces roughly the morphology of the $\mathrm{Al}_{2} \mathrm{O}_{3}(0001)$ substrate, and atomic height terraces are observed on the surface. On the other hand, a noticeable change is seen for Co films deposited at 300 and $600{ }^{\circ} \mathrm{C}$. In this case, the formation of nanoparticles with increasing diameter and height is observed. To obtain the nanoparticle diameter and mean interparticle distance (defined with respect to the centers of the nanoparticles), we used the 2D autocorrelation of the WSXM software. ${ }^{19}$

The nanoparticle average height was calculated by averaging the heights found in several AFM profiles. It should be mentioned that convolution effects of the microscope tip cannot be disregarded in the AFM images since the tip radius is of the same order of magnitude as the nanoparticle diameter. Moreover, the tip cannot penetrate the interspace between adjacent nanoparticles, and as a result, the measured height can be smaller than the actual one. The existence of a continuous film underneath the nanoparticles cannot also be neglected. Using this formalism, the Co film deposited at $300{ }^{\circ} \mathrm{C}$ was found to consist of nanoparticles with average diameter and height of 15 and $0.7 \mathrm{~nm}$, respectively, and av- 
TABLE I. Average diameter $(d)$, height $(h)$, and mean intercenter distance $(l)$ (all dimensions in $\mathrm{nm}$ ) obtained from the AFM images for the nanoparticles of the uncapped and $\mathrm{Al}, \mathrm{Au}$, and Pt capped systems, whose Co films were deposited at 300 and $600{ }^{\circ} \mathrm{C}$.

\begin{tabular}{|c|c|c|c|c|c|c|c|c|c|c|c|c|}
\hline & \multicolumn{3}{|c|}{$\mathrm{Co}$} & \multicolumn{3}{|c|}{$\mathrm{Co} / \mathrm{Al}$} & \multicolumn{3}{|c|}{$\mathrm{Co} / \mathrm{Au}$} & \multicolumn{3}{|c|}{$\mathrm{Co} / \mathrm{Pt}$} \\
\hline & $d$ & $l$ & $h$ & $d$ & $l$ & $h$ & $d$ & $l$ & $h$ & $d$ & $l$ & $h$ \\
\hline $300^{\circ} \mathrm{C}$ & 15 & 20 & 0.7 & 16 & 17 & 0.4 & 16 & 17 & 0.8 & 12 & 20 & 0.8 \\
\hline $600{ }^{\circ} \mathrm{C}$ & 17 & 29 & 2 & 15 & 25 & 0.5 & 14 & 25 & 0.7 & 14 & 27 & 1.1 \\
\hline
\end{tabular}

erage interparticle distance of $20 \mathrm{~nm}$. The Co film deposited at $600{ }^{\circ} \mathrm{C}$ was found to contain larger nanoparticles with average diameter and height of 17 and $2 \mathrm{~nm}$, respectively, and average interparticle distance of $29 \mathrm{~nm}$.

After establishing the Co growth mode as a function of the deposition temperature, the morphology of the capped Co films was investigated. As mentioned previously, $3.5 \mathrm{~nm}$ thick Al, Au, and Pt capping layers were deposited at RT in order to obtain a conformal growth mode with the Co films. AFM images for the Co films grown at RT, 300, and $600{ }^{\circ} \mathrm{C}$, and subsequently capped with $\mathrm{Al}, \mathrm{Au}$, and $\mathrm{Pt}$ are shown in Fig. 4(b). The measured diameter, height, and interparticle distance for these films, together with those of the uncapped samples, are listed in Table I. Regarding the Al capped systems [second row in Fig. 4(b)], the RT deposited film has a similar structure to the uncapped RT film. However, the Co films that were grown at 300 and $600{ }^{\circ} \mathrm{C}$ exhibit nanoparticles of similar size to that of the uncapped films, but with smaller heights. The reduction of the nanoparticle height may be due to the oxidation of the $\mathrm{Al}$ capping layer resulting in amorphous $\mathrm{Al}_{2} \mathrm{O}_{3}$, which has a tendency to fill the interparticle areas and thus smooth the surface. In the third row of Fig. 4(b), the AFM images of the Au capped systems are depicted. Again a flat surface reproducing the substrate atomic height terraces is observed in the Co film grown at RT. A slight reduction of the average nanoparticle height in the Co films grown at 300 and $600{ }^{\circ} \mathrm{C}$ is also found due to the tendency of Au to fill the interparticle areas. Finally, it can be seen that Pt appears to be the capping material with the best conformal growth on Co [fourth row in Fig. 4(b)]. Indeed, a comparison of the AFM images in Fig. 4(b) and the parameter values listed in Table I suggests that the Pt capped Co films have morphologies very similar to those of the uncapped Co films.

\section{MAGNETIC ANISOTROPY}

\section{A. Macroscopic magnetic properties}

The magnetic anisotropy and magnetization reversal processes in the $1.2 \mathrm{~nm}$ thick films were studied by performing polar and transverse Kerr loops at RT. Transverse Kerr loops were carried out in situ before and after the capping layer deposition in order to study the influence of the capping layer on the magnetic behavior. In Fig. 5, transverse Kerr loops for the uncoated Co films deposited at RT, 300, and $600{ }^{\circ} \mathrm{C}$ are depicted. These loops were measured along the easy (lines) and hard (symbols) in-plane axes. The onset of uniaxial in-plane magnetic anisotropy can be seen in the con- tinuous Co film deposited at RT due to the presence of monoatomic height terraces, as described in Sec. III. However, this anisotropy disappears in the nanoparticulate Co films deposited at 300 and $600{ }^{\circ} \mathrm{C}$ since the appearance of nanoparticles induces an extra configurational anisotropy which smears out the uniaxial counterpart. ${ }^{20}$ In the case of the Co film deposited at $300{ }^{\circ} \mathrm{C}$, a nonsaturated minor loop is observed. The small nanoparticles in this film have a size approaching the superparamagnetic volume for Co at RT $\left(\sim 200 \mathrm{~nm}^{3}\right)$. The shape of the loop reflects the weak exchange coupling between these quasisuperparamagnetic nanoparticles. On the other hand, in the case of the nanoparticulate Co film grown at $600{ }^{\circ} \mathrm{C}$, in which the nanoparticles were found to be larger (average diameter of $\sim 17 \mathrm{~nm}$ ) and thus above the superparamagnetic limit, a ferromagnetic loop is observed. In the case of the uncoated Co films, it was not possible to obtain polar loops due to the oxidation of $\mathrm{Co}$ when exposed to air.

The polar and transverse Kerr loops measured at RT for the capped Co films are shown in Figs. 6-8. In the case of Al capping, a very small influence in the transverse loops (left column of Fig. 6) is observed when compared to the un-

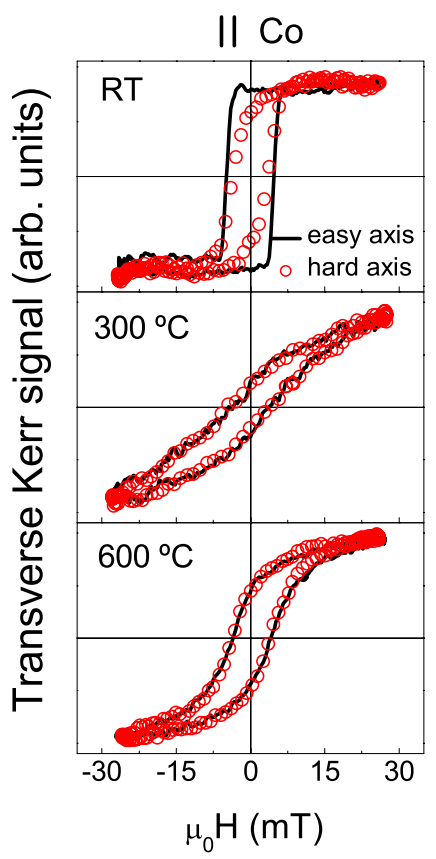

FIG. 5. (Color online) Transverse Kerr loops for the bare Co films deposited at RT, $300{ }^{\circ} \mathrm{C}$, and $600{ }^{\circ} \mathrm{C}$ measured with the applied magnetic field along the in-plane easy axis (black line) and hard axis (red dots). 
$\mathrm{II} \mathrm{Co} / \mathrm{Al} \perp \mathrm{Co} / \mathrm{Al}$

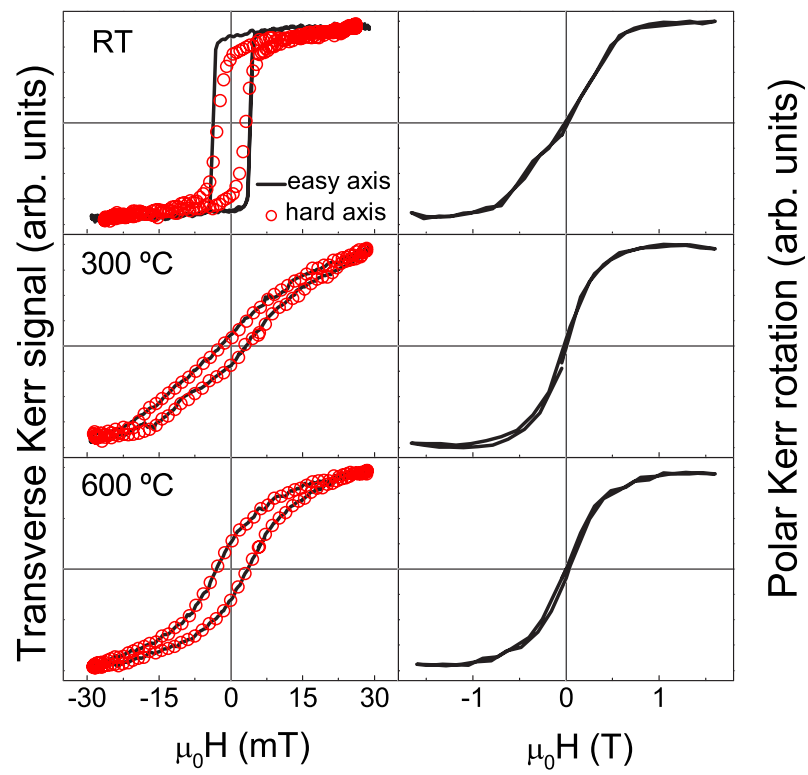

FIG. 6. (Color online) Left column: transverse Kerr loops for the $\mathrm{Al}$ capped $\mathrm{Co}$ films deposited at $\mathrm{RT}, 300{ }^{\circ} \mathrm{C}$, and $600{ }^{\circ} \mathrm{C}$ measured with the magnetic field along the in-plane easy axis (black line) and hard axis (red dots). Right column: corresponding polar Kerr loops measured with the applied magnetic field along the perpendicular direction. The Al capping does not appreciably affect the magnetic anisotropy of the system.

coated Co films (Fig. 5). Both the uniaxial anisotropy in the continuous film grown at RT and the above mentioned trend as a function of the nanoparticles size are clearly observed. Polar Kerr loops for the Al capped Co films are shown in the right column of Fig. 6. Closed loops with a high saturation

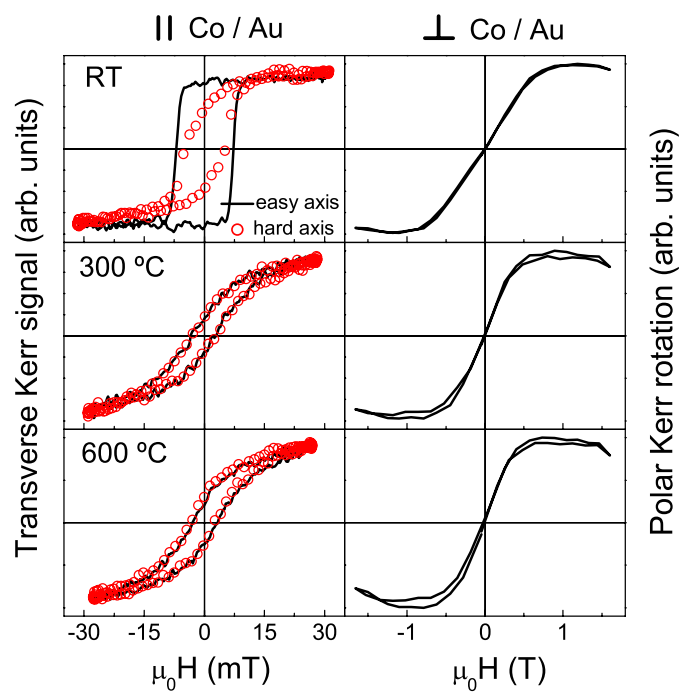

FIG. 7. (Color online) Left column: transverse Kerr loops for the Au capped Co films deposited at RT, $300{ }^{\circ} \mathrm{C}$, and $600{ }^{\circ} \mathrm{C}$ measured with the applied magnetic field along the in-plane easy axis (black line) and hard axis (red dots). Right column: corresponding polar Kerr loops measured with the applied magnetic field along the perpendicular direction.

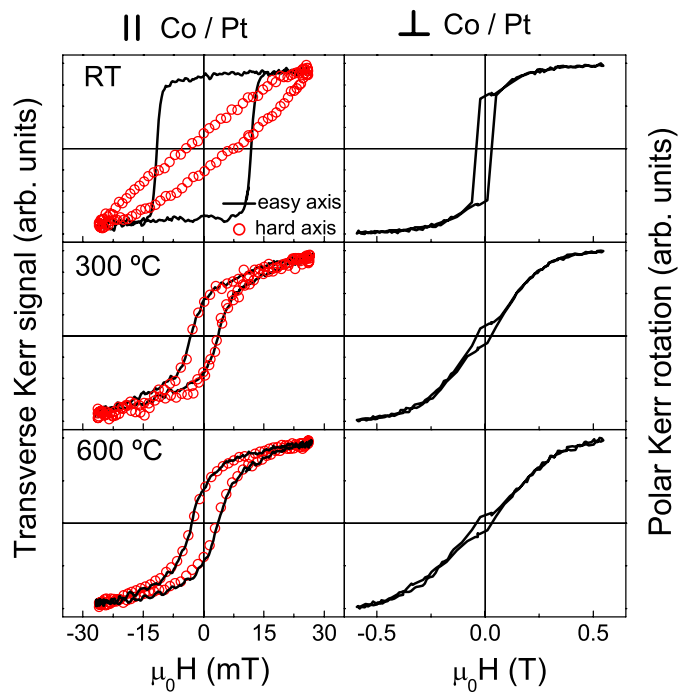

FIG. 8. (Color online) Left column: transverse Kerr loops for the Pt capped Co films deposited at RT, $300{ }^{\circ} \mathrm{C}$, and $600{ }^{\circ} \mathrm{C}$ measured with the applied magnetic field along the in-plane easy axis (black line) and hard axis (red dots). Right column: corresponding polar Kerr loops measured with the applied magnetic field along the perpendicular direction. A strong modification of the magnetic anisotropy is found due to the Pt polarization at the Pt-Co interface.

field are found in all cases, for systems in which the magnetization remains in-plane in equilibrium condition. The $2 \mathrm{D}$ to $3 \mathrm{D}$ growth mode transition is reflected by a decrease of the saturation field as the nanoparticles are formed and the demagnetization factor decreases.

For $\mathrm{Au}$ as the capping material, more dramatic modifications in the uniaxial magnetic anisotropy of the Co film grown at RT are found (left column of Fig. 7). An increase of the in-plane coercive and saturation fields is observed, related to an increase of the effective anisotropy constant $K_{\text {eff }}$. This change may be due either to the slight polarization of $\mathrm{Au}$ in the $\mathrm{Co} / \mathrm{Au}$ interface region or to the enhancement of the Co anisotropy, as observed in continuous and nanoparticulate films covered by Au. ${ }^{21,22}$ A very slight decrease of the saturation field can be observed in the nanoparticulate Co films grown at 300 and $600{ }^{\circ} \mathrm{C}$. In the case of the polar loops shown in the right column of Fig. 7, a more marked decrease of the saturation field related to the formation of nanoparticles is found, decreasing from $0.9 \mathrm{~T}$ in the continuous $\mathrm{Co}$ film to $0.7 \mathrm{~T}$ in the nanoparticulate $\mathrm{Co}$ film grown at $600{ }^{\circ} \mathrm{C}$.

Finally, the most pronounced effect is found in the $\mathrm{Pt}$ capped Co continuous and nanoparticulate films. The polarization of $\mathrm{Pt}$ when in contact with magnetic materials at $\mathrm{Co} / \mathrm{Pt}$ interfaces has been widely investigated by means of $\mathrm{XMCD},{ }^{6}$ Kerr spectroscopy, ${ }^{23,24}$ and $a b$ initio calculations. ${ }^{24,25}$ Polarization of the $\mathrm{Pt}$ atoms in contact with a Co surface is due to the hybridization of the Co $3 d$ and Pt $5 d$ orbitals. Suzuki et al. ${ }^{6}$ measured a magnetic moment per atom of $0.61 \mu_{B}$ for the Pt atoms at the interface, decreasing exponentially with the distance to the interface such that the first four atomic layers contain $90 \%$ of the total Pt magnetization. In addition, it has been found an increase of the perpendicular Co orbital moment localized at the $\mathrm{Co} / \mathrm{Pt}$ interface, giving rise to perpendicular magnetic anisotropy. ${ }^{26}$ 
As can be observed in Fig. 8, these effects strongly influence the magnetic anisotropy. For the $\mathrm{Pt}$ capped continuous Co film grown at RT (Fig. 8), a significant increase of the inplane coercive and saturation fields is found, being compatible with an increase of the in-plane effective magnetic anisotropy $K_{\text {eff }}$ In the case of the Co films grown at 300 and $600{ }^{\circ} \mathrm{C}$, a decrease of the saturation field is observed due to the presence of polarized $\mathrm{Pt}$ which is able to connect magnetically the nanoparticles, as found in earlier studies. ${ }^{7,8}$ In addition to the increase of in-plane magnetic anisotropy, the onset of a new magnetic anisotropy in the direction perpendicular to the surface is found in the polar loops (right column of Fig. 8). This is manifested as a strong decrease of the saturation field and the onset of hysteresis in the continuous Co film grown at RT (note the change of scale with respect to the $\mathrm{Al}$ and $\mathrm{Au}$ cases). In the case of the nanoparticulate films, a progressive weakening of the Pt polarization with increasing particle size is found, as demonstrated by the enhanced saturation field and reduced remanence. This fact is also supported by the MO spectral analysis discussed below. It is worth noting that in the case of the Pt capping, hysteresis is found in both the in-plane and perpendicular loops for a system with two combined anisotropies: on the one hand, the shape anisotropy of Co (both in continuous and nanoparticulate films) forces the magnetization to remain in plane, while on the other hand, the anisotropy induced by the $\mathrm{Co} / \mathrm{Pt}$ interface prefers the magnetization in the direction perpendicular to this interface.

\section{B. Atomic magnetic properties}

The XMCD technique is now well established in material science for the investigation of magnetic properties. In a magnetic material, the XMCD corresponds to the difference in X-ray absorption upon reversal of either the helicity of the circularly polarized photons or equivalently the magnetization direction of the sample. Since the absorption process involves transitions from atomic core levels to the spinpolarized valence band, the technique offers element selectivity, i.e., the ability to probe the magnetic properties of a chosen element in a complex material. The element selectivity of XMCD enables us to determinate the Co contribution in the magnetic behavior of the capped Co film structures discussed here.

Figure 9(a) displays the Co $L_{2,3}$ XAS for a reference sample with the structure $30 \AA \mathrm{Al} / 40 \AA \mathrm{Co} / \mathrm{Al}_{2} \mathrm{O}_{3}(0001)$ measured for opposite magnetization directions. The corresponding difference spectrum, i.e., the XMCD spectrum, is shown in Fig. 9(b). The Co magnetic moments were extracted from the XAS and XMCD spectra using the sum rules. ${ }^{27}$ Here, we have neglected in the analysis the so called saturation effects ${ }^{10,28}$ because these effects are expected to be negligible in thin film structures such as those studied here. The orbital moment $\mu_{L}$, spin moment $\mu_{S}$, magnetic dipole term $T_{z}$, and number of $3 d$ holes $n_{h}$ (all per atom) in the ground state are related to the integrated intensities of the difference $\Delta A_{2,3}$ and sum $A_{2,3}$ spectra over the corresponding $L_{2,3}$ edges according to ${ }^{27}$

$$
\frac{\mu_{L}}{n_{h}}=-\frac{4}{3} \frac{\Delta A_{3}+\Delta A_{2}}{A_{3}+A_{2}}
$$

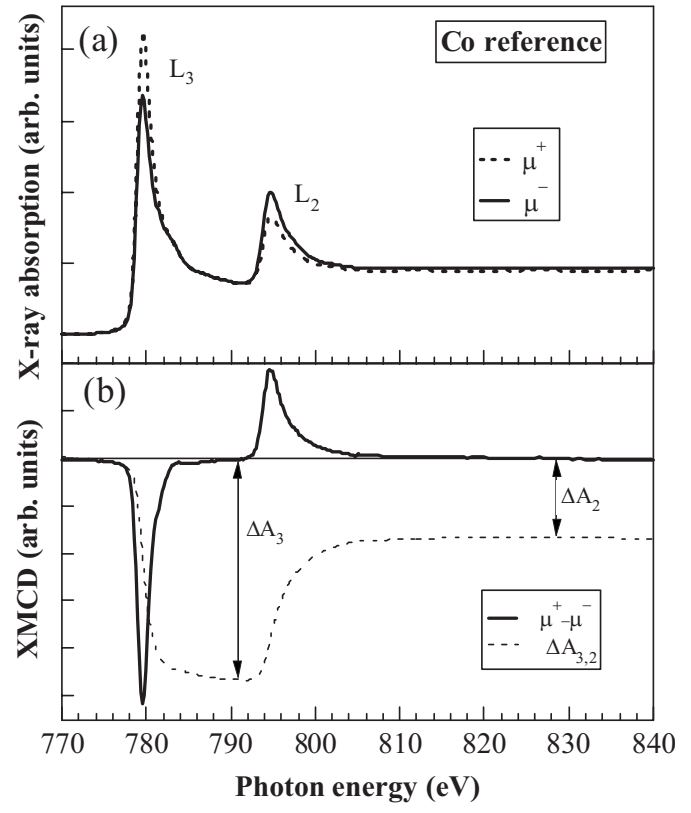

FIG. 9. Top panel: normalized XAS measured on the $30 \AA$ $\mathrm{Al} / 40 \AA \mathrm{Co} / \mathrm{Al}_{2} \mathrm{O}_{3}(0001)$ sample for the two opposite magnetizations. Bottom panel: corresponding difference spectrum (XMCD).

$$
\frac{\mu_{S}+7 T_{z}}{n_{h}}=-2 \frac{\Delta A_{3}-2 \Delta A_{2}}{A_{3}+A_{2}} .
$$

Theoretical results ${ }^{29,30}$ indicate that $7 T_{z} / \mu_{S}<0.1$, hence giving an error in $\mu_{S}$ up to $10 \%$ by neglecting $T_{z}$.

The effect of the capping layers and morphology on the magnetic moments is illustrated in Fig. 10. The XMCD spectra have been normalized to the Co $L_{3}$ peak and are also compared to the reference sample $30 \AA \quad \mathrm{Al} / 40 \AA$ $\mathrm{Co} / \mathrm{Al}_{2} \mathrm{O}_{3}(0001)$. It appears that $\mathrm{Al}$ and $\mathrm{Pt}$ capped samples produce a smaller area under the Co $L_{2}$ peak than the reference sample, which reflects an increased orbital moment contribution. Since the XMCD sum rule analysis returns the values of $\mu_{L} / n_{h}$ and $\mu_{S} / n_{h}$, knowledge of the exact number of $3 d$ holes is needed to obtain the values of the magnetic moments per atom. In low-dimensional structures, such as thin films and nanoparticles, the electronic structures are modified as compared to their "bulk" analogs. Such electronic structure modifications change the number of holes and consequently we cannot assume that $n_{h}$ for the thin films and nanoparticles studied here is the same as the bulk Co value. According to the XAS sum rule, ${ }^{31} n_{h}$ is proportional to $A_{3}+A_{2}$. Following the procedure used in previous work, ${ }^{32}$ the XAS spectra were normalized to the intensity above the $L_{2}$ edge and the relative change in the integrated XAS signal was determined for the different systems. The $30 \AA \mathrm{Al} / 40 \AA$ $\mathrm{Co} / \mathrm{Al}_{2} \mathrm{O}_{3}(0001)$ structure was taken as reference, assuming a value of $n_{h}=2.49$ for hep Co. ${ }^{33,34}$

The extracted values of $n_{h}$ (Table II) were found to be reduced from the bulk value by $\sim 10 \%$ as a consequence of the reduction of the Co film thickness. However, no significant influence of the nanostructure was found. The values of the atomic magnetic moments, taking into account the deviation of $n_{h}$ from the bulk Co value, are listed in Table III. 


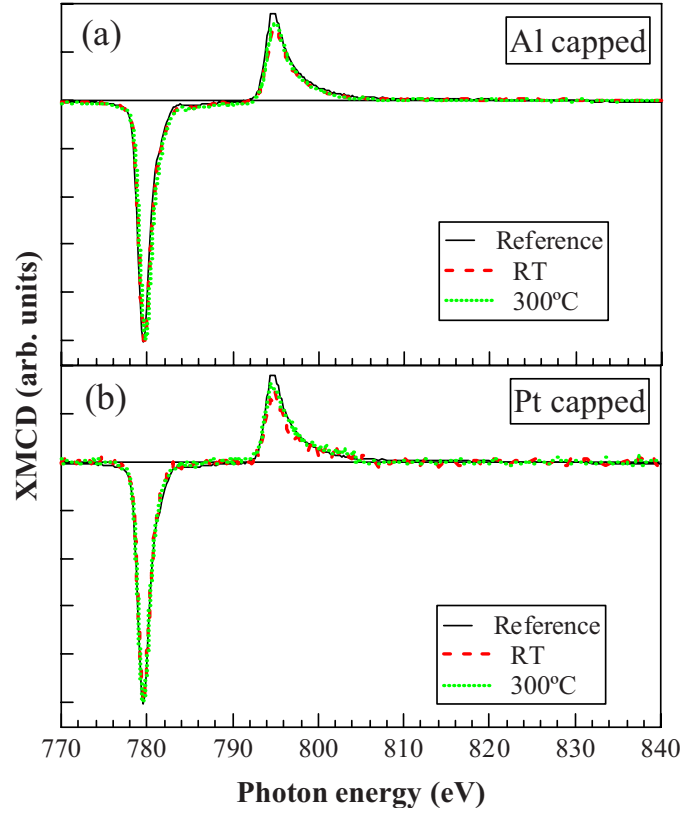

FIG. 10. (Color online) Normalized Co $L_{2,3}$ XMCD spectra showing the dependence on the capping layer and morphology of the deposited layers: Al capping (top panel) and Pt capping (bottom panel). The XMCD spectrum of the reference sample, $30 \AA$ $\mathrm{Al} / 40 \AA \mathrm{Co} / \mathrm{Al}_{2} \mathrm{O}_{3}(0001)$, is also shown for the purpose of comparison.

These values were obtained assuming that the mixing of the $j=3 / 2$ and $j=1 / 2$ states as well as the contribution of the $s p$ valence band to the $L_{2,3}$ XMCD signal can be neglected, and that $T_{z} \approx 0$.

From Table III, it can be seen that the Co films deposited at $300{ }^{\circ} \mathrm{C}$ possess higher spin and orbital atomic magnetic moments than those deposited at RT, independent of the capping composition. Hence, the nanostructuration produced by deposition at $300{ }^{\circ} \mathrm{C}$ has a dramatic effect on the atomic magnetic moments. However, the $\mu_{L} / \mu_{S}$ ratio of these nanostructured Co films was found to depend on the capping layer, reflecting its important role in controlling the magnetic properties. The $\mu_{L} / \mu_{S}$ ratio was increased for an Al capping and decreased for a Pt capping. This behavior suggests that the nanoparticles formed on the Co films deposited at $300{ }^{\circ} \mathrm{C}$ are not interconnected. Since $\mathrm{Al}$ has no $d$ states for hybridization with $\mathrm{Co}$, the magnetic interaction is small and the Al capped sample presented the typical response of isolated particles. ${ }^{4,5}$ On the other hand, the Pt capping magnetically shunts the particles due to the hybridization between Co and Pt and it reduces the $\mu_{L} / \mu_{S}$ ratio.

The effect of the capping layer was further analyzed by studying the atomic magnetic moments of the Co films de-
TABLE II. Number of $3 d$ hole $n_{h}$ depending on both the capping layers and the morphology of the deposits, assuming $n_{h}$ $=2.49$ for the reference sample.

\begin{tabular}{lcc}
\hline \hline Capping & Co RT & Co $300{ }^{\circ} \mathrm{C}$ \\
\hline $\mathrm{Pt}$ & $2.31(8)$ & $2.2(1)$ \\
$\mathrm{Al}$ & $2.17(2)$ & $2.13(2)$ \\
\hline \hline
\end{tabular}

posited at the same temperature. Compared to the reference sample, the atomic spin moment is enhanced with Pt capping and reduced with $\mathrm{Al}$ capping. Although both reference sample ( $4 \mathrm{~nm}$ Co thick) and the $1.2 \mathrm{~nm}$ Co thick sample were capped with $\mathrm{Al}$, it can be observed that they display different atomic magnetic moments. It is inferred that such behavior is related to the thickness of the Co layers. The effect of the formation of an interface alloy between the Co and the $\mathrm{Al}$ deposits is enhanced for the thinnest Co deposits when compared to the reference sample as also observed in the case of $\mathrm{Fe}$ nanoparticles capped with $\mathrm{Al} .{ }^{35}$

On the other hand, there is an enhancement of the atomic orbital moment for both $\mathrm{Pt}$ and $\mathrm{Al}$ cappings. This quantity is very sensitive to small changes in the surface structure ${ }^{36}$ and lowering the dimensionality is one of the main causes leading to an enhanced atomic orbital moment. ${ }^{37}$ In addition, this quantity also depends on the geometry, ${ }^{38}$ which could be the reason of the higher $\mu_{L}$ values obtained in the nanostructure samples, especially in the Al capped film. As a consequence of these variations, the Pt capped samples significantly enhance the total atomic magnetic moment, with the strongest effect observed in the Co film deposited at $300{ }^{\circ} \mathrm{C}$.

\section{MORPHOLOGY AND CAPPING EFFECTS IN THE MAGNETO-OPTICAL ACTIVITY}

MO Kerr spectroscopy is a very sensitive technique to the electronic structure of magnetic materials and to the nature of their environment. Consequently, it is also very sensitive to the morphology, to the matrix nature in the case of nanoparticles, and to the onset of magnetic polarization in the matrix material. To investigate the influence of the morphology and the matrix nature on the MO activity, polar Kerr rotation and ellipticity measurements were carried out in the optical region from 1.4 to $4.3 \mathrm{eV}$. Rotation and ellipticity spectra for $1.2 \mathrm{~nm}$ thick Co films grown at RT, $300{ }^{\circ} \mathrm{C}$, and $600{ }^{\circ} \mathrm{C}$ with capping layers of $3.5 \mathrm{~nm}$ of $\mathrm{Au}, \mathrm{Al}$, and $\mathrm{Pt}$ are shown in Figs. 11(a)-11(c), respectively. Very different trends as a function of the Co deposition temperature (and therefore morphology) are observed depending on the matrix material. For the Au capping, changes are observed between

TABLE III. Co atomic magnetic moments as a function of capping layers and morphology of the deposits.

\begin{tabular}{|c|c|c|c|c|c|c|c|c|c|}
\hline \multirow[b]{2}{*}{ Capping } & \multicolumn{3}{|c|}{ Co RT } & \multicolumn{3}{|c|}{ Co $300^{\circ} \mathrm{C}$} & \multicolumn{3}{|c|}{ Reference } \\
\hline & $\mu_{S}$ & $\mu_{L}$ & $\mu_{L} / \mu_{S}$ & $\mu_{S}$ & $\mu_{L}$ & $\mu_{L} / \mu_{S}$ & $\mu_{S}$ & $\mu_{L}$ & $\mu_{L} / \mu_{S}$ \\
\hline $\mathrm{Pt}$ & $1.89(3)$ & $0.23(1)$ & $0.12(1)$ & $2.35(3)$ & $0.266(6)$ & $0.11(1)$ & & & \\
\hline $\mathrm{Al}$ & $1.25(1)$ & $0.218(3)$ & $0.181(2)$ & $1.52(2)$ & $0.289(2)$ & $0.194(3)$ & $1.61(1)$ & $0.168(2)$ & $0.125(3)$ \\
\hline
\end{tabular}




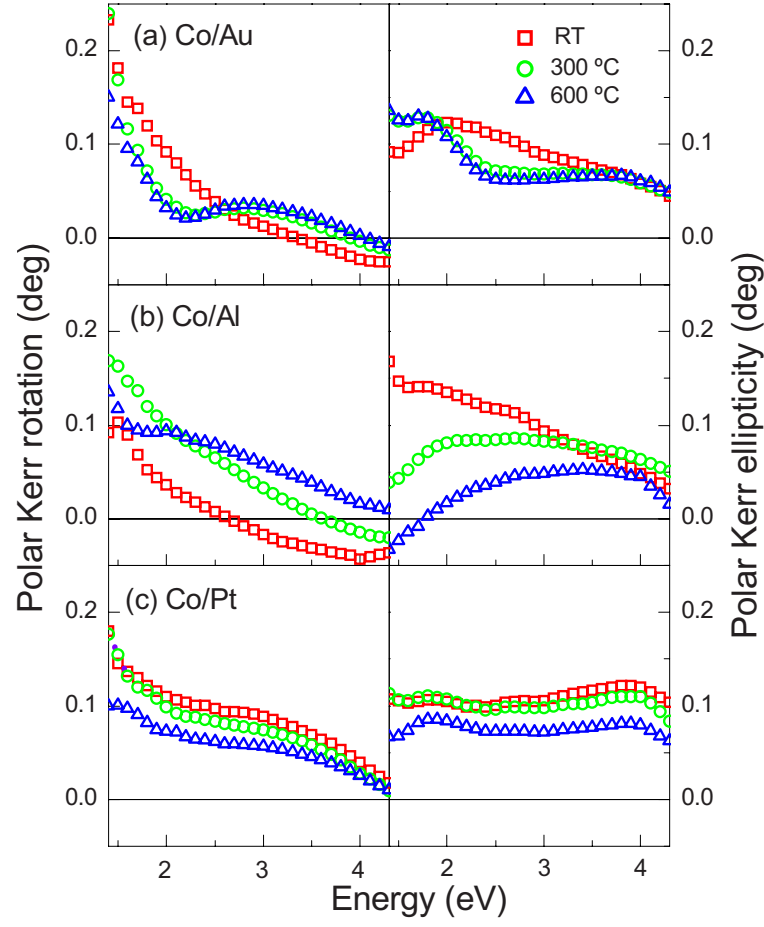

FIG. 11. (Color online) Polar Kerr rotation and ellipticity spectra for the Co films grown at RT, $300{ }^{\circ} \mathrm{C}$, and $600{ }^{\circ} \mathrm{C}$ and capped with (a) Au, (b) Al, and (c) Pt. The spectral trend depends strongly on the capping material.

the continuous film (with Co grown at RT) and the nanoparticulate films (with Co grown at 300 and $600{ }^{\circ} \mathrm{C}$ ), where a structure centered at $2.5 \mathrm{eV}$ appears in the latter case (inflection point in rotation and minimum in ellipticity). It is worth noticing that the spectra for the nanoparticulate Au-capped Co films are almost identical. A very different behavior is observed for the Al capped films [Fig. 11(b)], showing a progressive increase in rotation and decrease in ellipticity for increasing Co deposition temperature as nanoparticles are formed. Finally, Pt capped systems [Fig. 11(c)] show a progressive intensity decrease in rotation and ellipticity as nanoparticles are formed.

In order to explain this trend in the rotation and ellipticity spectra, numerical simulations were carried out by means of two different formalisms: the transfer matrix formalism ${ }^{39,40}$ and the scattering matrix formalism. ${ }^{41,42}$ The former describes the optical and MO response of systems consisting of a number of continuous layers stacked along the growth direction and is suitable to simulate the systems whose Co film was deposited at RT. Nevertheless, to describe the complex structure of the capped Co nanoparticulate films grown at 300 and $600{ }^{\circ} \mathrm{C}$, it was necessary to use the above mentioned scattering matrix formalism, which is able to describe properly the complex morphology of the capped nanoparticulate films and to calculate its optical and MO activity. Rotation and ellipticity spectra for the Au-capped continuous Co film grown at RT [Fig. 11(a)] were used to extract the MO constants of Co [which may differ from those of the bulk material due to its small thickness and to effects related to the presence of $\mathrm{Co} / \mathrm{Al}_{2} \mathrm{O}_{3}(0001)$ and $\mathrm{Co} / \mathrm{Au}$ interfaces]. For this purpose, the transfer matrix formalism was used,

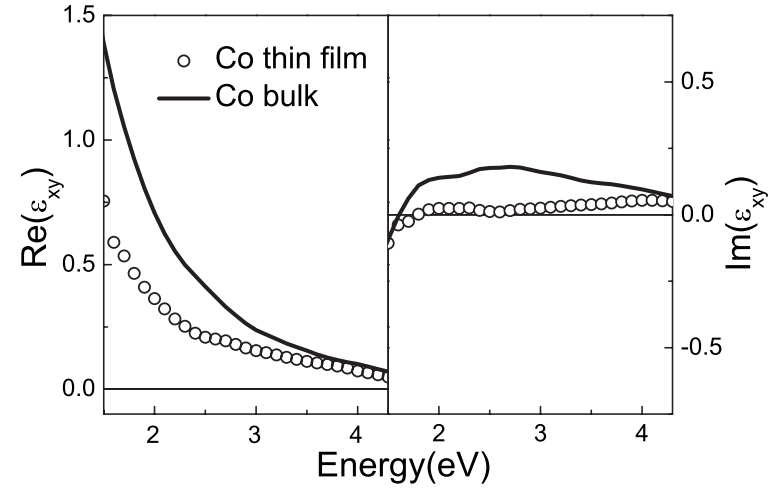

FIG. 12. Co MO constants extracted from the Au-capped continuous Co film, together with those obtained for bulk Co by Weller et al. (Ref. 44).

taking into account the $\mathrm{Co}$ and $\mathrm{Au}$ optical constants measured by ellipsometry. As previously mentioned, the Au polarization at the $\mathrm{Co} / \mathrm{Au}$ interface is an order of magnitude lower than of $\mathrm{Pt}$ at $\mathrm{Co} / \mathrm{Pt}$, having an atomic magnetic moments of $\sim 0.03 \mu_{B}$ (Ref. 43), and can therefore be neglected in the simulations. The MO constants obtained for the $\mathrm{Co}$ film are depicted in Fig. 12, together with those of bulk Co (Ref. 44) for comparison. It can be noticed that, compared to the bulk material, the Co MO constants in the continuous film are lower over the whole spectral range. These obtained MO constants were used in the subsequent simulations, presented below.

For the Au-capped nanoparticulate films whose Co film was grown at 300 and $600{ }^{\circ} \mathrm{C}$, it was necessary to use the scattering matrix formalism to simulate the rotation and ellipticity spectra. The structure of these nanoparticulate systems was modeled by dividing them into several layers along the growth direction and by considering the nanoparticles as forming an in-plane triangular array. In Fig. 13(a), the cross sectional morphology of the two nanoparticulate systems is sketched, taking into account the data obtained from the analysis of the AFM images. The corresponding models considered for the simulations (cross sections and plain views) are shown in Fig. 13(b). The dimensions of the cylinders and the thickness of the different layers forming the nanoparticles were selected according to the AFM observations. It is worth noticing that the AFM tip radius $(10 \mathrm{~nm})$ is of the same order as the interparticle distance, and therefore the measured nanoparticles height may be slightly smaller. Due to this fact, it was necessary to consider nanoparticles of 1.5 and $2.7 \mathrm{~nm}$ height for the nanoparticulate films with Co grown at 300 and $600{ }^{\circ} \mathrm{C}$, respectively, in order to obtain consistent results. It was also necessary to consider continuous Co films between the nanoparticles, with thicknesses of 7 and $5 \AA$ for the films with Co grown at 300 and $600{ }^{\circ} \mathrm{C}$, respectively. As can be observed in Fig. 13(c), using this model and the scattering matrix formalism, it was possible to reproduce the trend in the rotation and ellipticity spectra. Obviously, a perfect agreement was found for the sample where the Co film was grown at RT since it was used to extract the MO constants of Co. In the case of the nanoparticulate systems, whose Co film was grown at 300 and $600{ }^{\circ} \mathrm{C}$, an excellent agreement is obtained, reproducing the structure around 
(a)

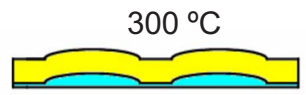

(b)

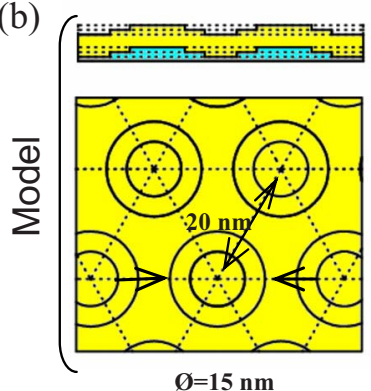

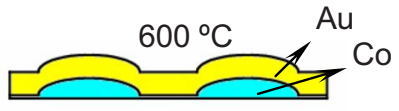
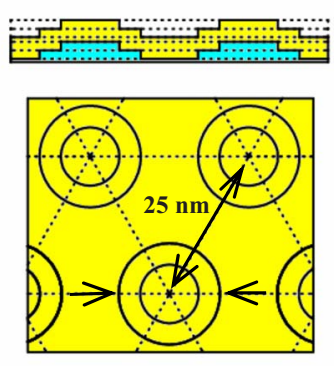

$\varnothing=17 \mathrm{~nm}$ (c)

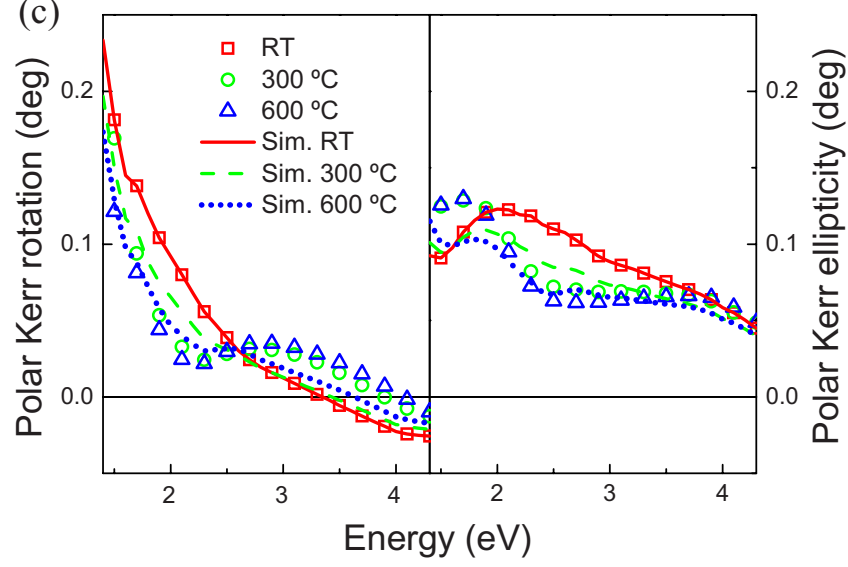

FIG. 13. (Color online) (a) Cross sectional sketches, deduced from the AFM data, of the $\mathrm{Au}$ capped systems where the Co films were grown at 300 and $600{ }^{\circ} \mathrm{C}$. (b) Cross section and plain views of the models considered in the simulations. (c) Experimental (symbols) and simulated (lines) polar Kerr rotation and ellipticity spectra corresponding to these systems.

$2.5 \mathrm{eV}$. It was inferred that this structure, which appears only in the nanoparticulate systems whose Co nanoparticles are surrounded by the Au matrix, is related to the Au plasmon resonance and represents a combined matrix and nanostructuration effect in the MO activity of this system.

Regarding the $\mathrm{Al}$ capped systems, a very different trend in the MO activity is observed, as seen in Fig. 11(b). As previously mentioned, $\mathrm{Al}$ oxidizes outside the vacuum, giving rise to an amorphous $\mathrm{Al}_{2} \mathrm{O}_{3}$ passivation layer on the surface with a fixed thickness that prevents further oxidization. This alteration strongly modifies the propagation of light in the system due to the onset of a transparent film on top and may also modify the MO activity since the electronic confinement conditions inside the nanoparticles embedded in a dielectric matrix change, as has been previously described for similar nanoparticulate systems embedded in dielectric matrix. ${ }^{45-47}$ In the case of the Al-capped Co continuous film grown at RT, it was deduced that in order to reproduce the rotation and ellipticity spectra, with the MO constants for Co presented in Fig. 12, it is necessary to take a $3 \AA$ thick metallic Al layer on Co, capped by a $3.2 \mathrm{~nm}$ thick $\mathrm{Al}_{2} \mathrm{O}_{3}$ passivation layer, as sketched in Fig. 14(a). In the case of the nanoparticulate films, simulations were performed using the scattering matrix formalism and modeling their structure analogously to (a)
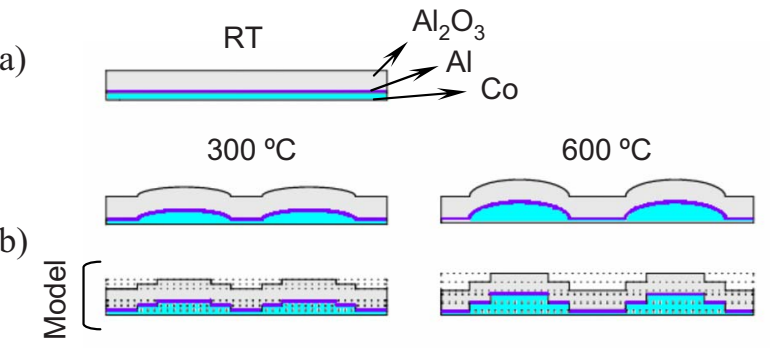

(c)

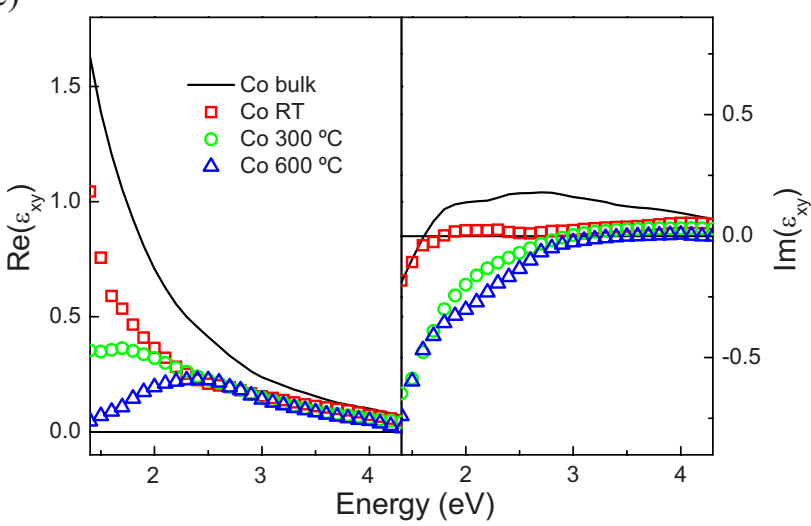

FIG. 14. (Color online) (a) Cross sectional sketches, deduced from the AFM data, of the Al capped systems where the Co films were grown at RT, $300{ }^{\circ} \mathrm{C}$, and $600{ }^{\circ} \mathrm{C}$. (b) Cross section of the models considered in the simulations. (c) MO constants of the Co present in these systems.

the Au capped systems, supposing in this case a $3 \AA$ thick metallic Al film on the Co nanoparticles followed by a $3.2 \mathrm{~nm}$ thick $\mathrm{Al}_{2} \mathrm{O}_{3}$ film, as sketched in Fig. 14(b). In this case, it was not possible to reproduce the trend in the rotation and ellipticity spectra by only changing the morphology of the systems. This suggests that the dielectric tensor of Co may be different to that of the continuous Co film due to the mentioned confinement effects. In order to investigate this point, the scattering matrix formalisms and the rotation and ellipticity spectra were used to extract the Co MO constants in the systems with $\mathrm{Co}$ films grown at $\mathrm{RT}, 300^{\circ} \mathrm{C}$, and $600{ }^{\circ} \mathrm{C}$. The calculated MO constants are depicted in Fig. 14(c), together with the bulk Co MO constants ${ }^{44}$ for comparison. Strong variations can be observed between them, with a progressive decrease in the intensity as the nanostructuration is more evident, which is compatible with the observed progressive decrease in the electronic relaxation time, associated with confinement effects inside the nanoparticles. ${ }^{45-47}$ It is therefore demonstrated that the $\mathrm{Al}$ capping after oxidation acts as a dielectric matrix, leading to strong matrix effects in the MO activity of the system.

Finally, the influence of the Pt capping layer on the MO activity of the systems was investigated. As shown in Fig. 11(c), a progressive intensity decrease is observed in both rotation and ellipticity spectra as the nanostructuration is more evident. In the case of the continuous Pt capped Co film, simulations were performed by means of the transfer matrix formalism to simulate the rotation and ellipticity spectra. Taking into account the MO constants calculated for Co (Fig. 12) and considering no Pt polarization at the inter- 
(a)

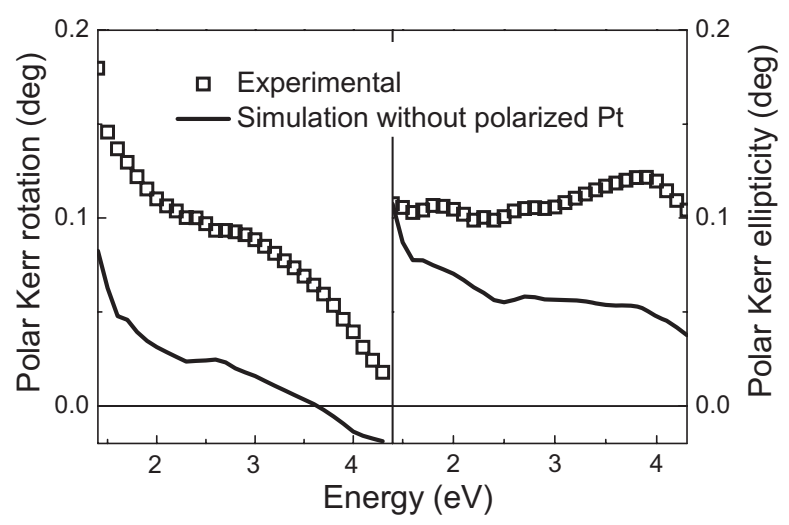

(b)

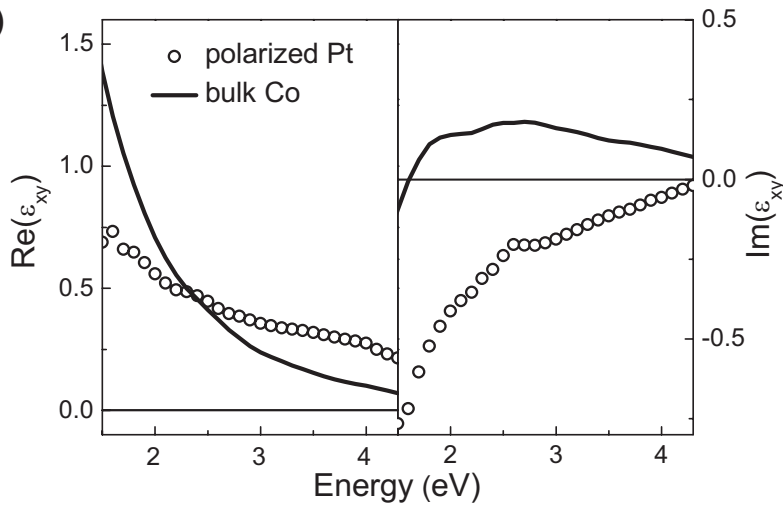

FIG. 15. (a) Experimental and simulated polar Kerr spectra for the Pt capped continuous Co film, considering no Pt polarization at the $\mathrm{Co} / \mathrm{Pt}$ interface. (b) MO constants calculated for the polarized Pt film, together with those obtained for the bulk material for comparison.

face, the intensity of the obtained rotation and ellipticity spectra is much lower than the experiment [Fig. 15(a)]. This fact reveals the necessity to consider a Pt polarized film at the $\mathrm{Co} / \mathrm{Pt}$ interface and allows us to extract the MO constants of such a film from the measured rotation and ellipticity spectra. As previously mentioned, Pt polarization at the $\mathrm{Co} / \mathrm{Pt}$ interface decreases exponentially with the distance, in such a way that the first four atomic layers contain $90 \%$ of the Pt magnetization. ${ }^{6}$ In this case, as in previous work, ${ }^{7}$ we considered a 3.5 atomic layers thick $(\sim 7 \AA)$ polarized Pt film at the $\mathrm{Co} / \mathrm{Pt}$ interface, with a constant magnetic moment throughout its entire thickness. ${ }^{7,8}$ Adding this polarized Pt film and using the matrix transfer formalism, it was possible to extract the $\mathrm{MO}$ constant of polarized $\mathrm{Pt}$ from the rotation and ellipticity spectra of the above mentioned sample, and they are shown in Fig. 15(b) together with the MO constants of bulk Co (Ref. 44) for comparison. It is worth noticing that the real parts are always positive and decrease with the energy, whereas a sign change is observed in the imaginary part. Nevertheless, the thickness of the Pt polarized film may depend on the thickness of the Co film below it. To investigate this point a $5 \AA$ thick Co film capped with the same thickness of Pt $(3.5 \mathrm{~nm})$ was prepared. From the analysis of its rotation and ellipticity spectra, it was inferred that the Pt polarized film in this case is reduced to 1.5 atomic layers ( $3 \AA$ ). This fact may affect the total amount of polarized Pt (a)
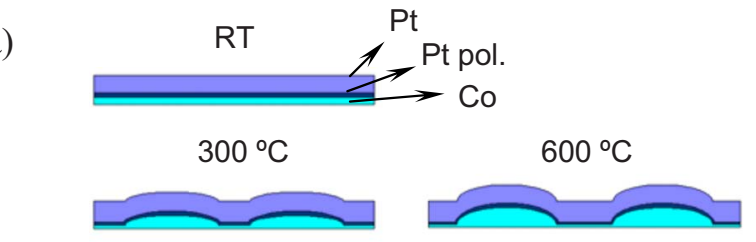

(b)

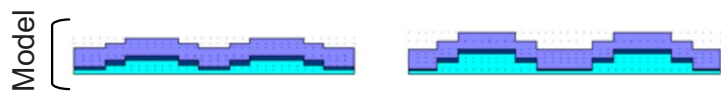

(c)

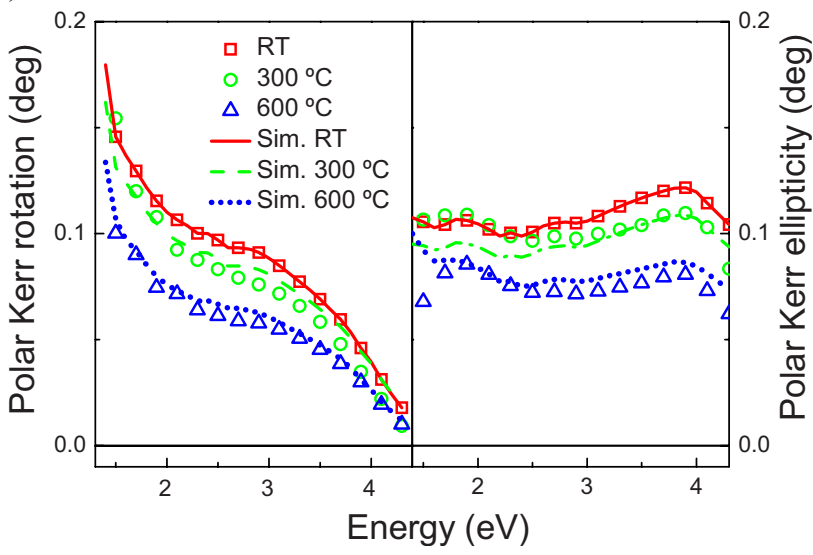

FIG. 16. (Color online) (a) Cross sectional sketches, deduced from the AFM data, of the Pt capped systems where the Co films were grown at RT, $300{ }^{\circ} \mathrm{C}$, and $600{ }^{\circ} \mathrm{C}$. (b) Cross sections of the models considered in the simulations. (c) Experimental (symbols) and simulated (lines) polar Kerr rotation and ellipticity spectra corresponding to these systems.

in the case of the nanoparticulate systems with Co films grown at 300 and $600{ }^{\circ} \mathrm{C}$ since the thickness of Co in the interparticle areas is lower than $1.2 \mathrm{~nm}$.

The scattering matrix formalism was used to perform the simulations in these systems, considering the same morphology for $\mathrm{Co}$ as in previous cases, since their deposition conditions were identical. In this case, polarized Pt films were added onto Co [Fig. 16(a)], with a thickness of $7 \AA$ on top of the nanoparticles, since their height is larger than $1.2 \mathrm{~nm}$, and a lower thickness of 3.5 and $2 \AA$ in the interparticle areas for the system with Co films grown at 300 and $600{ }^{\circ} \mathrm{C}$, respectively. This reduction of the amount of polarized $\mathrm{Pt}$ as the Co deposition temperature increases leads to a progressive decrease of the intensity in both the rotation and ellipticity spectra, as the experimental results reflect. As can be observed in Fig. 16(c), an excellent agreement is obtained between experimental data and simulations, which demonstrates that the Co morphology leads to polarization effects in the Pt film that modify the global MO activity of the system.

\section{CONCLUSIONS}

We have studied the influence of the morphology and capping layer polarization on the magnetic and magneto-optical properties of nanostructured Co films. It was found that the preparation of films at 300 and $600{ }^{\circ} \mathrm{C}$ promoted the growth of nanoparticles with increasing height and diameter. The 
formation of nanoparticles promoted a change in magnetic anisotropy from purely in plane to partially out of plane, as evidenced by the decrease of the perpendicular saturation field. The influence of the nanoparticles was also observed in measurements of the Co atomic magnetic moment, where enhanced spin and orbital contributions were found in samples prepared at elevated temperatures.

In addition to morphology effects, the capping layer was also found to play a crucial role in the overall magnetic properties. The use of a nonpolarizable $\mathrm{Al}$ capping displayed little influence on the magnetic behavior of the system. The nanostructures grown at $300{ }^{\circ} \mathrm{C}$ demonstrated a superparamagnetic response as a consequence of a weak exchange coupling. The enhancement of the orbital to spin magnetic moment ratio measured with XMCD confirmed the individual response of these structures. However, more dramatic effects on the magnetic response of the whole system were witnessed when magnetically polarized capping layers were used. In particular, the use of a Pt capping layer leads to an increase in the effective in-plane magnetic anisotropy of the system. In the case of the nanoparticulate films, the Pt was able to magnetically shunt the nanoparticles via exchange interactions and this suppressed the superparamagnetic character that was observed for the $\mathrm{Al}$ capped samples.

Apart from the Pt contribution to the magnetic response of the whole system, the Co-Pt hybridization also modifies the Co magnetic properties. The use of the element-specific XMCD technique made it possible to isolate the Co magnetic contribution from the system as a whole. Compared to the films capped with $\mathrm{Al}$, the $\mathrm{Pt}$ capping enhanced the total $\mathrm{Co}$ atomic magnetic moment. This enhancement was particularly strong in the case of the nanostructured films. The collective behavior of the Co nanostructures due to the Pt capping was also evidenced by a decrease of the Co $3 d$ orbital to spin magnetic moment ratio, $\mu_{L} / \mu_{S}$, compared to the $\mathrm{Al}$ capped system. Thus, it can be concluded that the use of a polarizable capping layer enhances the magnetic properties of the system not only by the polarization of Pt layers at the interface but also by an enhancement of the Co atomic magnetic moment.

In this work, we found a clear correlation between the trend of the atomic magnetic moments measured for Co with $\mathrm{XMCD}$ and the collective magnetic response of each system measured by Kerr techniques. The combination of both techniques enables to give a global overview of how the magnetic response of the system is driven by the Co atomic magnetic moments. In addition, this combination also provides information about how the capping layer not only modifies the individual magnetic response of Co atoms but also its own contribution to the global magnetic response in terms of the degree of polarization.

Further evidence of the influence of morphology and capping layer on the MO properties was found via measurements of the Kerr rotation and ellipticity spectra and successfully reproduced by numerical simulations using the scattering matrix formalism. Effects related to surface plasmon resonance in the case of the Au capped films and to electronic confinement inside the Co nanoparticles in the case of the Al capping layer are found. Finally, an enhancement of the global MO activity is found in the Pt capped systems due to Pt polarization, being observed a reduction in the nanoparticulate films due to morphology effects.

\section{ACKNOWLEDGMENTS}

We acknowledge the SRS staff for the support during the XMCD experiment. C.C., L.M., and Y.H. acknowledge the Spanish Ministerio de Educación y Ciencia and the Consejo Superior de Investigaciones Científicas (CSIC) for financial support through FPI, "Juan de La Cierva," and "Ramón y Cajal" programes, respectively. The CAM under the program NANOMAGNET S-0505/MAT/0194, Ministerio de Educación y Ciencia under Project Nos. MAT2005-05524C02-01 and NAN2004-09195-C04-01, and CSIC under Project No. $200650 I 130$ are also acknowledged.
${ }^{1}$ J. P. Bucher, D. C. Douglass, and L. A. Bloomfield, Phys. Rev. Lett. 66, 3052 (1991).

${ }^{2}$ S. Linderoth and S. N. Khanna, J. Magn. Magn. Mater. 104, 1574 (1992).

${ }^{3}$ S. E. Apsel, J. W. Emmert, J. Deng, and L. A. Bloomfield, Phys. Rev. Lett. 76, 1441 (1996).

${ }^{4}$ H. A. Dürr, G. van der Laan, J. Vogel, G. Panaccione, N. B. Brookes, E. Dudzik, and R. McGrath, Phys. Rev. B 58, R11853 (1998).

${ }^{5}$ H. A. Dürr, S. S. Dhesi, E. Dudzik, D. Knabben, G. van der Laan, J. B. Goedkoop, and F. U. Hillebrecht, Phys. Rev. B 59, R701 (1999).

${ }^{6}$ M. Suzuki, H. Muraoka, Y. Inaba, H. Miyagawa, N. Kawamura, T. Shimatsu, H. Maruyama, N. Ishimatsu, Y. Isohama, and Y. Sonobe, Phys. Rev. B 72, 054430 (2005).

${ }^{7}$ E. Navarro, Y. Huttel, C. Clavero, G. Armelles, and A. Cebollada, Appl. Phys. Lett. 84, 2139 (2004).

${ }^{8}$ E. Navarro, Y. Huttel, C. Clavero, A. Cebollada, and G. Armelles,
Phys. Rev. B 69, 224419 (2004).

${ }^{9}$ Y. Huttel, H. Gómez, C. Clavero, A. Cebollada, G. Armelles, E. Navarro, M. Ciria, L. Benito, J. I. Arnaudas, and A. J. Kellok, J. Appl. Phys. 96, 1666 (2004).

${ }^{10}$ R. Nakajima, J. Stöhr, and Y. U. Idzerda, Phys. Rev. B 59, 6421 (1999).

${ }^{11}$ W. S. Kim, M. Aderholz, and W. Kleemann, Meas. Sci. Technol. 4, 1275-1280 (1993).

${ }^{12}$ H. J. Choi, Z. Q. Qiu, J. Pearson, J. S. Jiang, Dongqi Li, and S. D. Bader, Phys. Rev. B 57, R12713 (1998).

${ }^{13}$ J. Chen and J. L. Erskine, Phys. Rev. Lett. 68, 1212 (1992).

${ }^{14}$ A. Berger, U. Linke, and H. P. Oepen, Phys. Rev. Lett. 68, 839 (1992).

${ }^{15}$ W. Weber, A. Bischof, R. Allenspach, C. H. Back, J. Fassbender, U. May, B. Schirmer, R. M. Jungblut, G. Güntherodt, and B. Hillebrands, Phys. Rev. B 54, 4075 (1996).

${ }^{16}$ W. Weber, C. H. Back, A. Bischof, Ch. Würsch, and R. Allenspach, Phys. Rev. Lett. 76, 1940 (1996). 
${ }^{17}$ R. K. Kawakami, E. J. Escorcia-Aparicio, and Z. Q. Qiu, Phys. Rev. Lett. 77, 2570 (1996).

${ }^{18}$ J. Hamrle, J. Ferré, J. P. Jamet, V. Repain, G. Baudot, and S. Rousset, Phys. Rev. B 67, 155411 (2003).

${ }^{19}$ I. Horcas, R. Fernandez, J. M. Gomez-Rodriguez, J. Colchero, J. Gomez-Herrero, and A. M. Baro, Rev. Sci. Instrum. 78, 013705 (2007).

${ }^{20}$ F. Cebollada, A. Hernando-Mañeru, A. Hernando, C. MartínezBoubeta, A. Cebollada, and J. M. Gonzalez, Phys. Rev. B 66, 174410 (2002).

${ }^{21}$ F. Luis, F. Bartolomé, F. Petroff, J. Bartolomé, L. M. García, C. Deranlot, H. Jaffrès, M. J. Martínez, P. Bencok, F. Wilhelm, A. Rogalev, and N. B. Brookes, Europhys. Lett. 76, 142 (2006).

${ }^{22}$ D. Weller, J. Stöhr, R. Nakajima, A. Carl, M. G. Samant, C. Chappert, R. Megy, P. Beauvillain, P. Veillet, and G. A. Held, Phys. Rev. Lett. 75, 3752 (1995).

${ }^{23}$ C. Train, P. Beauvillain, V. Mathet, G. Pénissard, and P. Veillet, J. Appl. Phys. 86, 3165 (1999).

${ }^{24}$ Y. P. Lee, K. W. Kim, R. Gontarz, and Y. V. Kudryavtsev, Curr. Appl. Phys. 1, 451 (2001).

${ }^{25}$ S. Uba, L. Uba, A. N. Yaresko, A. Ya. Perlov, V. N. Antonov, and R. Gontarz, Phys. Rev. B 53, 6526 (1996).

${ }^{26}$ N. Nakajima, T. Koide, T. Shidara, H. Miyauchi, H. Fukutani, A. Fujimori, K. Iio, T. Katayama, M. Nyvlt, and Y. Suzuki, Phys. Rev. Lett. 81, 5229 (1998).

${ }^{27}$ B. T. Thole, P. Carra, F. Sette, and G. van der Laan, Phys. Rev. Lett. 68, 1943 (1992); P. Carra, B. T. Thole, M. Altarelli, and X. Wang, ibid. 70, 694 (1993); C. T. Chen, Y. U. Idzerda, H.-J. Lin, N. V. Smith, G. Meigs, E. Chaban, G. H. Ho, E. Pellegrin, and F. Sette, ibid. 75, 152 (1995).

${ }^{28}$ R. Nakajima, Ph.D. thesis, Stanford University, 1998.

${ }^{29}$ J. Stöhr and H. König, Phys. Rev. Lett. 75, 3748 (1995).

${ }^{30}$ H. A. Dürr and G. van der Laan, Phys. Rev. B 54, R760 (1996).

${ }^{31}$ B. T. Thole and G. van der Laan, Phys. Rev. A 38, 1943 (1988).

${ }^{32}$ S. S. Dhesi, H. A. Dür, G. van der Laan, E. Dudzik, and N. B. Brookes, Phys. Rev. B 60, 12852 (1999).
${ }^{33}$ R. Wu, D. Wang, and A. J. Freeman, Phys. Rev. Lett. 71, 3581 (1993); R. Wu and A. J. Freeman, ibid. 73, 1994 (1994).

${ }^{34}$ C. T. Chen, Y. U. Idzerda, H.-J. Lin, N. V. Smith, G. Meigs, E. Chaban, G. H. Ho, E. Pellegrin, and F. Sette, Phys. Rev. Lett. 75, 152 (1995).

${ }^{35}$ Y. Huttel, E. Navarro, N. D. Telling, G. van der Laan, F. Pigazo, F. J. Palomares, C. Quintana, E. Roman, G. Armelles, and A. Cebollada (unpublished).

${ }^{36}$ F. May, M. Tischer, D. Arvanitis, M. Russo, J. H. Dunn, H. Henneken, H. Wende, R. Chauvistre, N. Martensson, and K. Baberschke, Phys. Rev. B 53, 1076 (1996).

${ }^{37}$ M. Tischer, O. Hjortstam, D. Arvanitis, J. H. Dunn, F. May, K. Baberschke, J. Trygg, J. M. Wills, B. Johansson, and O. Eriksson, Phys. Rev. Lett. 75, 1602 (1995).

${ }^{38}$ R. A. Guirado-López and J. M. Montejano-Carrizales, Phys. Rev. B 75, 184435 (2007).

${ }^{39}$ M. Schubert, Phys. Rev. B 53, 4265 (1996).

${ }^{40}$ M. Schubert, T. E. Tiwald, and J. A. Woolam, Appl. Opt. 38, 177 (1999).

${ }^{41}$ D. M. Whittaker and I. S. Culshaw, Phys. Rev. B 60, 2610 (1999).

${ }^{42}$ A. García-Martín, G. Armelles, and S. Pereira, Phys. Rev. B 71, 205116 (2005).

${ }^{43}$ F. Wilhelm, M. Angelakeris, N. Jaouen, P. Poulopoulos, E. Th. Papaioannou, Ch. Mueller, P. Fumagalli, A. Rogalev, and N. K. Flevaris, Phys. Rev. B 69, 220404(R) (2004).

${ }^{44}$ D. Weller, G. R. Harp, R. F. C. Farrow, A. Cebollada, and J. Sticht, Phys. Rev. Lett. 72, 2097 (1994).

${ }^{45}$ C. Clavero, A. Cebollada, G. Armelles, Y. Huttel, J. Arbiol, F. Peiró, and A. Cornet, Phys. Rev. B 72, 024441 (2005).

${ }^{46}$ C. Clavero, B. Sepúlveda, G. Armelles, Z. Konstantinovic, M. García del Muro, A. Labarta, and X Batlle, J. Appl. Phys. 100, 074320 (2006).

${ }^{47}$ C. Clavero, G. Armelles, J. Mangueritat, J. Gonzalo, M. García del Muro, A. Labarta, and X. Batlle, Appl. Phys. Lett. 90, 182506 (2007). 